\title{
Prévalence du syndrome métabolique et de ses facteurs de risque chez les enfants et les adolescents canadiens : Enquête canadienne sur les mesures de la santé, cycle 1 (2007-2009) et cycle 2 (2009-2011)
}

M. MacPherson, M.B.A. (1,2); M. de Groh, Ph. D. (1); L. Loukine, M. Sc. (3); D. Prud'homme, M.D. (4,5); L. Dubois, Ph. D. (6)

Cet article a fait l'objet d'une évaluation par les pairs.

\section{Résumé}

Introduction : Nous avons étudié la prévalence du syndrome métabolique (SMet) et de ses facteurs de risque ainsi que l'influence du statut socioéconomique chez les enfants et les adolescents canadiens.

Méthodologie : Nous avons inclus dans notre étude les 1228 répondants de l'Enquête canadienne sur les mesures de la santé, cycle 1 (2007-2009) et cycle 2 (2009-2011), âgés de 10 à 18 ans et ayant fourni un échantillon de sang à jeun. Nous avons utilisé les définitions consensuelles du SMet proposées par la Fédération internationale du diabète (FID) pour les enfants et adolescents (10 à 15 ans) et pour les adultes (16 ans et plus). Nous avons mesuré la prévalence du SMet et de ses facteurs de risque ainsi que les différences en fonction du statut socioéconomique au moyen de tests du $\chi^{2}$.

Résultats : La prévalence du SMet était de 2,1 \% . Le tiers (37,7 \%) des répondants présentaient au moins un facteur de risque, les plus répandus étant l'obésité abdominale (21,6\%), un faible taux de C-HDL (19,1\%) et un taux de triglycérides élevé (7,9\%). Cette combinaison d'obésité abdominale, de faible taux de C-HDL et de taux élevé de triglycérides correspondait à $61,5 \%$ des cas de SMet. Les participants des ménages de la tranche supérieure de revenu et bénéficiant d'un niveau de scolarité élevé présentaient la plus faible prévalence d'un ou de plusieurs facteurs de risque du SMet, d'obésité abdominale et de faible taux de C-HDL.

Conclusion : La prévalence du SMet $(2,1 \%)$ s'est révélée inférieure à celle mesurée auparavant pour le Canada (3,5 \%) et les États-Unis (4,2 \% à 9,2 \%), sans doute en raison de l'application stricte des critères de la FID pour l'étude du SMet. Le tiers des enfants et des adolescents canadiens présentaient au moins un facteur de risque de SMet. Comme le risque de SMet augmente avec l'âge, ces estimations de la prévalence, couplées à une prévalence nationale de l'obésité d'environ $10 \%$ chez les jeunes, laissent entrevoir un accroissement du risque de SMet et d'autres maladies chroniques chez les jeunes Canadiens.

Mots-clés : Enquête canadienne sur les mesures de la santé, syndrome métabolique, enquêtes sur la santé, facteurs de risque cardiométabolique, prévalence, adolescents, enfants
Principales constatations

- Avoir le syndrome métabolique (SMet) augmente le risque d'avoir une maladie chronique : risque double pour une maladie cardiovasculaire et quintuple pour le diabète de type 2 .

- Seulement 2,1 \% des jeunes Canadiens souffrent du SMet. Cependant, un tiers ont un ou plusieurs facteurs de risque du SMet.

- Le plus important facteur de risque du SMet est l'obésité abdominale. Puisque de plus en plus de jeunes sont obèses, on s'attend à ce que le SMet augmente chez les jeunes Canadiens.

- Le risque du SMet augmente avec l'âge, donc le risque de maladies chroniques augmentera probablement avec le vieillissement de la population canadienne.

- Les jeunes des ménages avec un meilleur revenu ou un meilleur niveau de scolarité des parents ont le risque de SMet le plus faible.

\section{Introduction}

Les maladies chroniques constituent la principale cause de décès évitables au Canada et dans le monde, ainsi que le plus lourd fardeau évitable pour le système public de soins de santé $^{1}$. Le syndrome métabolique (SMet) est

Rattachements des auteurs :

1. Direction des déterminants sociaux et de l'intégration scientifique, Agence de la santé publique du Canada, Ottawa (Ontario), Canada

2. Faculté des études supérieures et postdoctorales, Université d'Ottawa, Ottawa (Ontario), Canada

3. Centre de prévention et de contrôle des maladies chroniques, Agence de la santé publique du Canada, Ottawa (Ontario), Canada

4. Institut de recherche de l'Hôpital Montfort, Ottawa (Ontario), Canada

5. Faculté des sciences de la santé, Université d’Ottawa, Ottawa (Ontario), Canada

6. Département d'épidémiologie et de médecine sociale, Faculté de médecine, Université d’Ottawa, Ottawa (Ontario), Canada

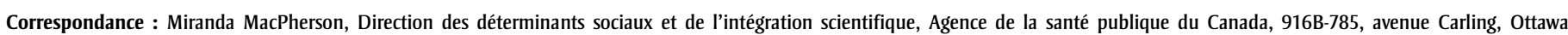
(Ontario) K1A 0K9; tél. : 613-668-4018; téléc. : 613-960-0921; courriel : miranda.macpherson@phac-aspc.ǵc.ca 
une constellation de facteurs de risque cardiométabolique prédicteurs de maladies chroniques et de mortalité, toutes causes confondues $^{2-4}$. On estime que les risques de maladies cardiovasculaires (MCV) doublent et que le risque de diabète de type 2 est quintuplé en présence du SMet ${ }^{3-6}$.

Le SMet est caractérisé par la présence de différentes combinaisons de facteurs de risque parmi les suivants : obésité, taux élevé de triglycérides à jeun, hypertension, insulinorésistance, taux élevé de cholestérol à lipoprotéines de faible densité, faible taux de cholestérol à lipoprotéines de haute densité (C-HDL), faible taux de cholestérol total, apolipoprotéine B élevée, protéine C-réactive élevée et homocystéine élevée ${ }^{7-9}$. Ces caractéristiques cliniques du SMet, lorsqu'elles sont réunies, suggèrent une étiologie commune: les hypothèses sur les mécanismes sous-jacents au SMet et leur influence sur les résultats en santé sont abordées ailleurs ${ }^{7,10,11}$.

La prévalence mondiale de l'obésité et du diabète a augmenté de façon significative au cours des 25 dernières années ${ }^{12}$, ce qui en retour a contribué à accroître la prévalence du SMet ${ }^{13}$. À l'échelle mondiale, la prévalence du SMet varie entre $1,2 \%$ et $22,6 \%$ chez les jeunes et entre $9,0 \%$ et $35,0 \%$ chez les adultes, en fonction de la définition du SMet retenue, de la région, de la conception de l'étude, de l'année de réalisation de l'étude, du groupe d'âge et de la populationcible $^{13-16}$. Au Canada, on estime que la prévalence du SMet chez l'adulte se situe entre $11,4 \%$ et $22,2 \%$, ce qui est supérieur aux estimations de $10 \%$ à $15 \%$ réalisées au début des années $1990^{17-22}$. À titre comparatif, la prévalence du SMet chez les adultes américains se situe entre $22 \%$ et $34 \%^{23-26}$. On considère que de manière générale la prévalence du SMet augmente significativement avec l'âge ${ }^{17-20,23}$. La prévalence chez les jeunes de 12 à 19 ans est de 3,5\% au Canada (d'après une étude menée en 2012 utilisant les critères du Adult Treatment Panel III pour le SMet) et de $4,2 \%$ à $9,2 \%$ aux É.-U., où entre $42 \%$ et $63 \%$ des jeunes présentent un ou plusieurs facteurs de risque de SMet ${ }^{19,27-29}$. Un examen plus poussé de la prévalence à l'échelle du Canada chez les jeunes va nous permettre de mieux comprendre la progression du SMet et de ses facteurs de risque au sein de la population.
De nombreuses données probantes font état d'une relation inversée entre statut socioéconomique (SSÉ) et MCV, ces dernières partageant certains facteurs de risque avec le $\mathrm{SMet}^{30-32}$. Les études axées sur le lien entre SSÉ et SMet révèlent un profil similaire: chez les individus de statut social inférieur, la prévalence du SMet est considérablement plus importante ${ }^{17,19,20,33,34}$. Les études canadiennes menées à l'échelle du pays ont montré que la prévalence du SMet est beaucoup moins élevée chez les membres des ménages à scolarité postsecondaire que chez ceux des ménages à niveau de scolarité inférieur, particulièrement chez les femmes ${ }^{17,19,20}$. Cette relation inversée est également valable entre revenu du ménage et SMet, quoique de manière moins prononcée : les ménages canadiens situés dans les quartiles inférieurs de revenu présentent une prévalence supérieure de SMet par rapport aux ménages dont le revenu est moyen ou supérieur $^{17,20}$.

L'un des défis associé à la mesure de la prévalence du SMet est l'existence de plusieurs critères et de plusieurs définitions pour le diagnostic de cette affection. C'est pourquoi la Fédération internationale du diabète (FID) a fait de la définition consensuelle du syndrome métabolique de la FID (IDF Consensus Worldwide Definition of the Metabolic Syndrome) un outil universellement reconnu et accepté ${ }^{35}$. Pour la FID, Le SMet implique la présence d'une obésité abdominale (mesurée par le tour de taille) et de deux facteurs de risque ou plus parmi les suivants : faible taux de C-HDL, hypertension, taux élevés de triglycérides à jeun et glycémie élevée ${ }^{6,36}$. Auparavant, les définitions les plus usuelles étaient celles de l'Organisation mondiale de la santé, de l'European Group for the Study of Insulin Resistance et du National Cholesterol Education Program Expert Panel on Detection, Evaluation, and Treatment of High Blood Cholesterol in Adults (critères du Adult Treatment Panel III) (NCEP ATP III) $)^{9,37,38}$.

Le diagnostic de SMet est particulièrement complexe chez les enfants et les adolescents, étant donné la difficulté à établir des critères précis, valables et harmonisés de ce syndrome. De ce fait, les estimations de la prévalence du SMet chez les enfants et les jeunes varient grandement en fonction de la définition retenue ${ }^{8}$. En 2007, la FID a publié sa définition consensuelle du syndrome métabolique chez les enfants et les adolescents (Consensus Definition of the Metabolic Syndrome in Children and Adolescents), en fonction de l'âge et du sexe, pour les jeunes de 10 à 15 ans $^{36}$. La FID recommande d'appliquer la définition internationale de l'adulte aux personnes de 16 ans et plus et de ne pas diagnostiquer de SMet chez les enfants de moins de 10 ans $^{36}$.

Notre étude visait principalement à examiner la prévalence du SMet et de ses facteurs de risque et à étudier l'influence du SSÉ sur ces facteurs de risque chez les enfants et les adolescents canadiens (10 à 18 ans) à l'aide de données nationales représentatives tirées de l'Enquête canadienne sur les mesures de la santé (ECMS). Elle met à profit une analyse nationale antérieure axée sur les jeunes Canadiens :

- en incluant les jeunes âgés de 10 et de 11 ans;

- en calculant la prévalence d'un ou de plusieurs facteurs de risque du SMet chez les jeunes;

- en examinant les modalités d'apparition des risques;

- en utilisant les données provenant des deux cycles de l'ECMS ${ }^{19}$.

Cette étude est la première étude nationale à appliquer rigoureusement la définition consensuelle du SMet chez les enfants et les adolescents proposée par la FID, qui est aujourd'hui la définition la plus récente et la plus universellement acceptée du SMet chez les jeunes. Elle est également la première étude à utiliser des données de référence sur l'âge et sur le tour de taille en fonction du sexe pour mesurer l'obésité abdominale chez les enfants et les adolescents canadiens.

\section{Méthodologie}

\section{Source des données}

L'ECMS est une enquête réalisée par Statistique Canada représentative à l'échelle nationale et conçue afin de recueillir de l'information sur la santé de la population 
canadienne ${ }^{39-41}$. Elle est axée sur des entrevues à domicile et sur des examens médicaux réalisés dans un centre d'examen mobile (CEM). Les entrevues permettent de recueillir des données sur les individus, leur SSÉ et leur histoire familiale ainsi que des informations générales sur leur santé. L'examen physique, pour sa part, comprend des mesures de l'anthropométrie, de la spirométrie, de la tension artérielle, de la forme physique et de la santé buccodentaire. Il inclut la collecte d'échantillons biologiques $^{39-41}$. L'enquête porte sur la population canadienne vivant à domicile, dans les 10 provinces et les 3 territoires. Elle est représentative de $96,3 \%$ de la population canadienne $e^{39-41}$. Sont exclus les personnes vivant dans des réserves ou dans d'autres établissements autochtones, celles vivant en institution ou dans certaines régions éloignées ainsi que les membres à temps plein des Forces canadiennes ${ }^{39-41}$. Le cycle 1 de l'ECMS (2007-2009) a recueilli des données auprès de la population âgée de 6 à 79 ans, tandis que le cycle 2 (2009-2011) en a élargi la portée à la population âgée de 3 à 79 ans $^{39-41}$.

L'ECMS offre des estimations fiables à l'échelle nationale par groupe d'âge et par sexe grâce à une stratégie d'échantillonnage en grappes ${ }^{39-42}$. Le choix des sites de collecte de données repose sur le registre de l'Enquête sur la population active. On a tenté de réduire au minimum, de multiples manières, les non-réponses: le taux de réponse combiné pour les visites à domicile et en clinique a été de $51,7 \%$ pour le cycle 1 et de $55,5 \%$ pour le cycle $2^{39-42}$. Statistique Canada a calculé le poids d'échantillonnage en multipliant le poids de sélection liés aux sites de collecte par les poids de sélection liés aux ménages, et en apportant une série d'ajustements pour les non-réponses à l'étape initiale, à l'étape des entrevues et à l'étape des examens dans les $\mathrm{CEM}^{42}$.

\section{Population visée par l'étude}

Les 1228 répondants à l'ECMS âgés de 10 à 18 ans ayant fourni des échantillons de sang à jeun dans le cadre du cycle 1 (2007-2009) ou du cycle 2 (2009-2011) ont été inclus dans l'étude. Aucune des participantes n'était enceinte. Un poids d'échantillonnage spécifique à ce sous-groupe ayant fourni des échantillons à jeun a été fourni par Statistique Canada afin d'assurer sa représentativité à l'échelle de l'ensemble de la population.

\section{Critères de diagnostic du SMet}

Nous avons appliqué les définitions consensuelles du SMet proposées par la FID : celle pour les enfants et les adolescents aux participants de 10 à 15 ans et celle pour les adultes aux participants de 16 à 18 ans. On a considéré que les enfants et adolescents étaient atteints de SMet en présence d'obésité abdominale (tour de taille égal ou supérieur à celui du $90^{\mathrm{e}}$ centile, en fonction de l'âge et du sexe) et de deux caractéristiques cliniques ou plus parmi les suivantes : taux élevé de triglycérides $(\geq 1,7 \mathrm{mmol} / \mathrm{L})$, faible taux de C-HDL $(<1,03 \mathrm{mmol} / \mathrm{L})$, tension artérielle élevée (tension artérielle systolique $\geq 130 \mathrm{~mm} \mathrm{Hg}$ ou tension artérielle diastolique $\geq 85 \mathrm{~mm} \mathrm{Hg}$ ou diagnostic d'hypertension) et glycémie élevée ( $\geq 5,6 \mathrm{mmol} / \mathrm{L}$ ou diagnostic de diabète de type 2$)^{36}$. On a considéré que les adultes étaient atteints de SMet en présence d'obésité abdominale et de deux caractéristiques cliniques ou plus parmi les suivantes : taux élevé de triglycérides $(\geq 1,7 \mathrm{mmol} / \mathrm{L})$, faible taux de C-HDL $(<1,03 \mathrm{mmol} / \mathrm{L}$ chez l'homme et $<1,29 \mathrm{mmol} / \mathrm{L}$ chez la femme), tension artérielle élevée (TA systolique $\geq 130 \mathrm{~mm} \mathrm{Hg}$ ou $\mathrm{TA}$ diastolique $\geq 85 \mathrm{~mm} \mathrm{Hg}$ ou diagnostic d'hypertension) et glycémie élevée $(\geq 5,6 \mathrm{mmol} / \mathrm{L}$ ou diagnostic de diabète de type 2$)^{35}$.

Nous avons défini l'obésité abdominale à partir des données de référence sur le tour de taille du $90^{\mathrm{e}}$ centile en fonction de l'âge et du sexe de l'Enquête sur la condition physique au Canada de $1981^{43}$. Nous avons appliqué les seuils calculés pour les enfants de 11 ans à ceux âgés de 10 et de 11 ans car n'étaient fournies des estimations que pour les jeunes de 11 à 18 ans.

\section{Variables liées aux caractéristiques individuelles et au statut socioéconomique}

Les caractéristiques individuelles et le SSÉ des répondants ont été évalués au moyen de variables sur le niveau de scolarité associé au ménage, sur le revenu du ménage, sur l'identité autochtone et sur le statut vis-à-vis de l'immigration. Nous avons utilisé les mêmes variables sur le niveau de scolarité et sur le revenu du ménage que dans les études antérieures examinant la relation entre SSÉ et SMet $^{17,19,20,26}$. La scolarité est l'indicateur de SSÉ le plus utilisé dans les études épidémiologiques et celui qui tend à avoir le lien le plus fort et le plus systématique avec la santé cardiovasculaire $20,31,44$. Le revenu du ménage est également souvent utilisé pour rendre compte du SSÉ et constitue un déterminant de la santé ${ }^{44-48}$. Statistique Canada a calculé le revenu du ménage en classant les répondants en différentes catégories en fonction du revenu total de leur ménage et du nombre total de personnes dans leur ménage ${ }^{39,40}$.

Pour améliorer la pertinence statistique des données, nous avons reclassifié les variables associées au niveau de scolarité et au revenu du ménage en trois catégories au lieu de quatre. Nous avons fusionné les catégories «revenu faible» et «revenu intermédiaire inférieur » en une seule catégorie intitulée « revenu faible et intermédiaire inférieur » et nous avons maintenu les catégories « revenu intermédiaire supérieur » et « revenu élevé ». De même, nous avons fusionné les catégories «études secondaires non complétées » et «diplôme d'études secondaires » en une seule catégorie intitulée «diplôme d'études secondaires ou moins » et nous avons maintenu les catégories « études postsecondaires partielles » et « diplôme d'études postsecondaires ».

\section{Analyse statistique}

Nous avons réalisé les analyses statistiques au moyen du SAS, version 9.3 (SAS Institute Inc., Cary, Caroline du Nord, États-Unis) pour le traitement des données et l'estimation des écarts au moyen de la méthode bootstrap ${ }^{49}$. La prévalence du SMet et de chacun des facteurs de risque a été estimée et exprimée sous forme de fréquences et de pourcentages avec un intervalle de confiance (IC) à $95 \%$. Les différences au niveau du SMet et de chacun des facteurs en fonction du sexe, de l'identité autochtone et du statut vis-àvis de l'immigration du répondant et en fonction du niveau de scolarité et du revenu associés à son ménage ont été mesurées par des tests du $\chi^{2}$, en utilisant 
la pondération et la méthode bootstrap. Le seuil de signification statistique a été fixé à $p$ inférieure à 0,05 .

Nous avons obtenu l'approbation éthique pour ce projet du Comité d'éthique de la recherche de l'Université d'Ottawa.

\section{Résultats}

\section{Description de l'échantillon étudié}

Des 2707 enfants et adolescents de 10 à 18 ans de l'échantillon initial, nous n'avons inclus pour notre étude que ceux ayant fourni des échantillons de sang à jeun, soit 1228 participants, avec légèrement plus de garçons $(51,5 \%)$ que de filles (48,5\%). Le tableau 1 donne un aperçu de l'échantillon en fonction des caractéristiques individuelles des répondants et du SSÉ.

\section{Prévalence du SMet}

Seuls 25 participants à l'étude ont reçu un diagnostic de SMet, soit 2,1\% (IC à $95 \%$ : 0,8 à 3,3)* (tableau 2). En raison de ce faible effectif de participants souffrant du SMet, il était impossible d'effectuer une ventilation précise par sexe, par âge ou par SSÉ.

\section{Prévalence des facteurs de risque individuels}

Environ le tiers $(37,7 \%$; IC à $95 \%$ : 33,8 à 41,6) des enfants et adolescents évalués présentaient au moins l'une des caractéristiques cliniques du SMet (1 facteur de risque ou plus) (tableau 2). Par ordre de prévalence, ces facteurs de risque étaient : l'obésité abdominale (21,6\%; IC à $95 \%$ : 16,6 à 26,7), un faible taux de C-HDL $(19,1 \%$; IC à $95 \%$ : 16,6 à 21,8$)$, un taux élevé de triglycérides $(7,9 \%$; IC à $95 \%$ : $4,8$ à 11,0$)$ et une glycémie élevée $(1,7 \%$; IC à $95 \%: 0,7$ à 2,8 $)^{\dagger}$. La prévalence d'une tension artérielle élevée était trop faible pour permettre une estimation statistique précise. Il n'y avait aucune différence en fonction du sexe dans les prévalences de chacun des facteurs de risque.

TABLEAU 1

Profil de l'échantillon ${ }^{\mathrm{a}}$, sous-échantillon à jeun, 10-18 ans

\begin{tabular}{|c|c|c|}
\hline Caractéristiques & Échantillon de l'étude (n) & $\begin{array}{c}\text { Pourcentage de l'échantillon } \\
\text { de l'étude (\%) }\end{array}$ \\
\hline \multicolumn{3}{|l|}{ Caractéristiques individuelles $(n=1228)$} \\
\hline \multicolumn{3}{|l|}{ Sexe } \\
\hline Garçons & 632 & 51,5 \\
\hline Filles & 596 & 48,5 \\
\hline \multicolumn{3}{|l|}{ Âge (ans) } \\
\hline 10 & 172 & 14,0 \\
\hline 11 & 184 & 15,0 \\
\hline 12 & 127 & 10,3 \\
\hline 13 & 151 & 12,3 \\
\hline 14 & 115 & 9,4 \\
\hline 15 & 117 & 9,5 \\
\hline 16 & 131 & 10,7 \\
\hline 17 & 121 & 9,8 \\
\hline 18 & 110 & 9,0 \\
\hline \multicolumn{3}{|l|}{ Profil socioéconomique } \\
\hline \multicolumn{3}{|l|}{ Niveau de revenu $(n=1178)$} \\
\hline Inférieur et intermédiaire inférieur & 247 & 19,7 \\
\hline Intermédiaire supérieur & 333 & 25,8 \\
\hline Supérieur & 598 & 50,4 \\
\hline \multicolumn{3}{|l|}{ Scolarité du ménage ( $n=1193$ ) } \\
\hline $\begin{array}{l}\text { Diplôme d'études secondaires ou d'un } \\
\text { grade inférieur }\end{array}$ & 126 & 11,0 \\
\hline Études postsecondaires partielles & 81 & 6,4 \\
\hline Diplôme d'études postsecondaires & 986 & 78,3 \\
\hline \multicolumn{3}{|l|}{ Identité ou origine autochtone $(n=1227)$} \\
\hline Autochtone & 46 & 4,4 \\
\hline Non-Autochtone & 1181 & 95,5 \\
\hline \multicolumn{3}{|l|}{ Statut vis-à-vis de l'immigration } \\
\hline Immigrant & 120 & 10,2 \\
\hline Non-immigrant & 1108 & 89,8 \\
\hline
\end{tabular}

${ }^{a}$ Les chiffres s'appuient sur des données brutes.

\section{Profil de la combinaison des facteurs de risque}

Les facteurs de risque individuels les plus répandus étaient l'obésité abdominale $(10,7 \%)$, un faible taux de C-HDL $(9,8 \%)$ et un taux élevé de triglycérides $(2,7 \%)$ (tableau 3). Les combinaisons de deux facteurs de risque les plus répandues étaient l'obésité abdominale et un faible taux de C-HDL (5,1\%) et l'obésité abdominale et un taux élevé de triglycérides (1,5\%). La combinaison de trois facteurs de risque la plus répandue était l'obésité abdominale associée à un faible taux de C-HDL et à un taux de triglycérides élevé $(1,3 \%)$. Cette combinaison correspondait à 61,5\% des cas de SMet (tableau 3).

\section{Associations entre statut socioéconomique} (scolarité et revenu du ménage) et facteurs de risque

Les répondants des ménages aux revenus les plus élevés étaient ceux qui présentaient le moins de facteurs de risque simples ou combinés en termes de pourcentage (35,5\%; IC à $95 \%$ : 29,8 à 41,2), le moins

"Vu le coefficient de variation (CV) de 29,0 , ce résultat est publié sous toute réserve.

†En raison de la petite taille des effectifs, il n'a pas été possible de rendre compte de tous les facteurs de risque et de toutes les catégories de SSÉ. 
TABLEAU 2

Prévalence $^{\mathrm{a}}$ du syndrome métabolique et de ses facteurs de risque, sous-échantillon à jeun, $10-18$ ans $(\mathbf{n}=1228)$

\begin{tabular}{|c|c|c|c|c|c|c|c|}
\hline \multirow[t]{2}{*}{ Affection } & \multicolumn{2}{|c|}{ Échantillon total } & \multicolumn{2}{|c|}{ Homme } & \multicolumn{2}{|c|}{ Femme } & \multirow[t]{2}{*}{ Valeur $p$} \\
\hline & Fréquence (n) & $\begin{array}{c}\% \text { (IC à } 95 \%) \\
\text { CV }\end{array}$ & Fréquence (n) & $\begin{array}{c}\% \text { (IC à } 95 \%) \\
\text { CV }\end{array}$ & Fréquence (n) & $\begin{array}{c}\% \text { (IC à } 95 \%) \\
\text { CV }\end{array}$ & \\
\hline SMet & 25 & $\begin{array}{c}2,1(0,8 \text { à } 3,3) \\
0,29^{b}\end{array}$ & - & - & - & - & - \\
\hline $\begin{array}{l}\text { Présence de } 2 \text { facteurs de risque } \\
\text { ou plus }\end{array}$ & 123 & $\begin{array}{c}10,8 \text { (7,4 à } 14,2) \\
0,15\end{array}$ & 71 & $\begin{array}{c}6,1(3,5 \text { à } 8,7) \\
0,02\end{array}$ & 52 & $\begin{array}{c}4,7(2,8 \text { à } 6,5) \\
0,19\end{array}$ & 0,3658 \\
\hline $\begin{array}{l}\text { Présence d'un facteur de risque } \\
\text { ou plus }\end{array}$ & 420 & $\begin{array}{c}37,7(33,8 \text { à } 41,6) \\
0,05\end{array}$ & 212 & $\begin{array}{c}18,1\left(\begin{array}{l}(15,4 \text { à } 20,8) \\
0,07\end{array}\right.\end{array}$ & 208 & $\begin{array}{c}19,6(16,4 \text { à } 22,9) \\
0,08\end{array}$ & 0,3179 \\
\hline Obésité abdominale & 240 & $\begin{array}{c}21,6(16,6 \text { à } 26,7) \\
0,11\end{array}$ & 130 & $\begin{array}{c}10,6 \text { (7,3 à } 13,9) \\
0,15\end{array}$ & 110 & $\begin{array}{c}11,0(7,5 \text { à } 14,5) \\
0,16^{\text {b }}\end{array}$ & 0,7443 \\
\hline Faible taux de C-HDL & 218 & $\begin{array}{c}19,1(16,6 \text { à } 21,8) \\
0,06\end{array}$ & 107 & $\begin{array}{c}8,8(6,6 \text { à } 11,0) \\
0,12\end{array}$ & 111 & $\begin{array}{c}10,54(8,4 \text { à } 12,3) \\
0,09\end{array}$ & 0,2863 \\
\hline Taux élevé de triglycérides & 82 & $\begin{array}{l}7,9(4,8 \text { à } 11,0) \\
0,19^{\mathrm{b}}\end{array}$ & 42 & $\begin{array}{c}4,7(2,2 \text { à } 7,3) \\
0,26^{\text {b }}\end{array}$ & 40 & $\begin{array}{c}3,2(1,7 \text { à } 7,2) \\
0,22^{\text {b }}\end{array}$ & \\
\hline
\end{tabular}

Abréviations : C-HDL, cholestérol à lipoprotéines de haute densité; $\mathrm{CV}$, coefficient de variation; IC, intervalle de confiance; TA, tension artérielle.

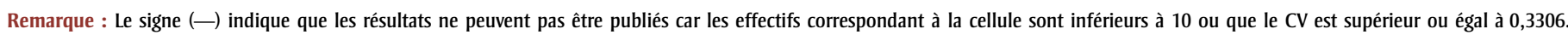
La prévalence d'une TA élevée était trop faible pour fournir une estimation statistique précise.

${ }^{a}$ Ces chiffres s'appuient sur des données pondérées.

${ }^{b}$ Ces chiffres sont publiés sous toute réserve puisque le CV était compris entre 0,16 et 0,33.

d'obésité abdominale $(18,4 \%$; IC à $95 \%$ : $11,7$ à 25,1$)$ et le plus faible taux de C-HDL $(17,5 \%$; IC à $95 \%$ : 14,2 à 20,6) par rapport aux familles ayant un revenu inférieur et intermédiaire inférieur (tableau 4). Les résultats relatifs à la scolarité montrent que les ménages comptant une personne détenant un diplôme d'études postsecondaires présentaient le moins de facteurs de risque simples ou combinés en termes de pourcentage (35,3\%; IC à $95 \%$ : 31,0 à 39,6), d'obésité abdominale (19,8\%; IC à $95 \%$ : 14,6 à 25,0) et de faible taux de C-HDL (17,5\%; IC $95 \%$ : 14,8 à 20,2) par rapport aux ménages associés à des études postsecondaires partielles ou à un diplôme d'études secondaires ou d'un grade inférieur. En raison de la petite taille des effectifs, les résultats n'ont pas pu être ventilés selon l'identité autochtone ou le statut vis-àvis de l'immigration.

\section{Analyse}

La prévalence du SMet chez les enfants et les adolescents $(2,1 \%)$ était inférieure à la prévalence mesurée pour l'ensemble du Canada $(3,5 \%)$ et pour les États-Unis $(4,2 \% \text { à } 9,2 \%)^{19,27-29}$. En présumant que notre échantillon est représentatif, cette prévalence de 2,1\% signifierait qu'environ 64832 enfants et adolescents sont touchés par le SMet au Canada. La prévalence d'un ou de plusieurs facteurs de risque $(37,7 \%)$ chez les enfants et les adolescents était également inférieure aux valeurs mesurées pour les États-Unis $(42 \% \text { à } 63 \%)^{28}$. La prévalence inférieure obtenue dans le cadre de notre étude par rapport aux estimations nationales antérieures visant la jeunesse canadienne peut être attribuable à l'utilisation de la définition du SMet proposée par la
FID, qui est fondée sur des critères légèrement plus rigoureux, en particulier sur la présence obligatoire d'obésité abdominale $\mathrm{e}^{19,43,50,51}$. Par ailleurs, on sait que le SMet augmente avec l'âge, et notre échantillon comprenait de nombreux enfants (10 et 11 ans) et davantage de jeunes (356 répondants de 10 et 11 ans) que d'adolescents (231 répondants de 17 et 18 ans) $)^{17,23}$.

TABLEAU 3

Profil d'association des facteurs de risque du syndrome métabolique ${ }^{\mathrm{a}}$

\begin{tabular}{|c|c|}
\hline Combinaison des facteurs de risque $(n=1228)$ & Fréquence (\%) \\
\hline \multicolumn{2}{|l|}{ Présence d'un facteur de risque } \\
\hline Obésité abdominale & $131(10,7)$ \\
\hline Faible C-HDL & $121(9,8)$ \\
\hline Taux élevé de TG & $33(2,7)$ \\
\hline \multicolumn{2}{|l|}{ Présence de deux facteurs de risque } \\
\hline Obésité abdominale + faible C-HDL & $63(5,1)$ \\
\hline Obésité abdominale + taux élevé de TG & $19(1,5)$ \\
\hline \multicolumn{2}{|l|}{ Présence de trois facteurs de risque } \\
\hline Obésité abdominale + faible C-HDL + taux élevé de TG & $16(1,3)$ \\
\hline Association de facteurs de risque chez les répondants souffrant du SMet $(n=26)$ & Fréquence $(\%)$ \\
\hline \multicolumn{2}{|l|}{ Présence de trois facteurs de risque } \\
\hline Obésité abdominale + faible C-HDL + taux élevé de TG & $16(61,5)$ \\
\hline
\end{tabular}

Abréviations : C-HDL, cholestérol à lipoprotéines de haute densité; TG, triglycérides.

Remarque : Les associations de facteurs de risque portant sur des effectifs inférieurs à 10 n'ont pas été publiées car la prévalence était trop faible pour permettre une estimation statistique exacte.

${ }^{a}$ Ces chiffres sont fondés sur des données pondérées. 
TABLEAU 4

Liens entre les facteurs de risque du syndrome métabolique et le statut socioéconomique ${ }^{\mathrm{a}}$, sous-échantillon à jeun, 10-18 ans

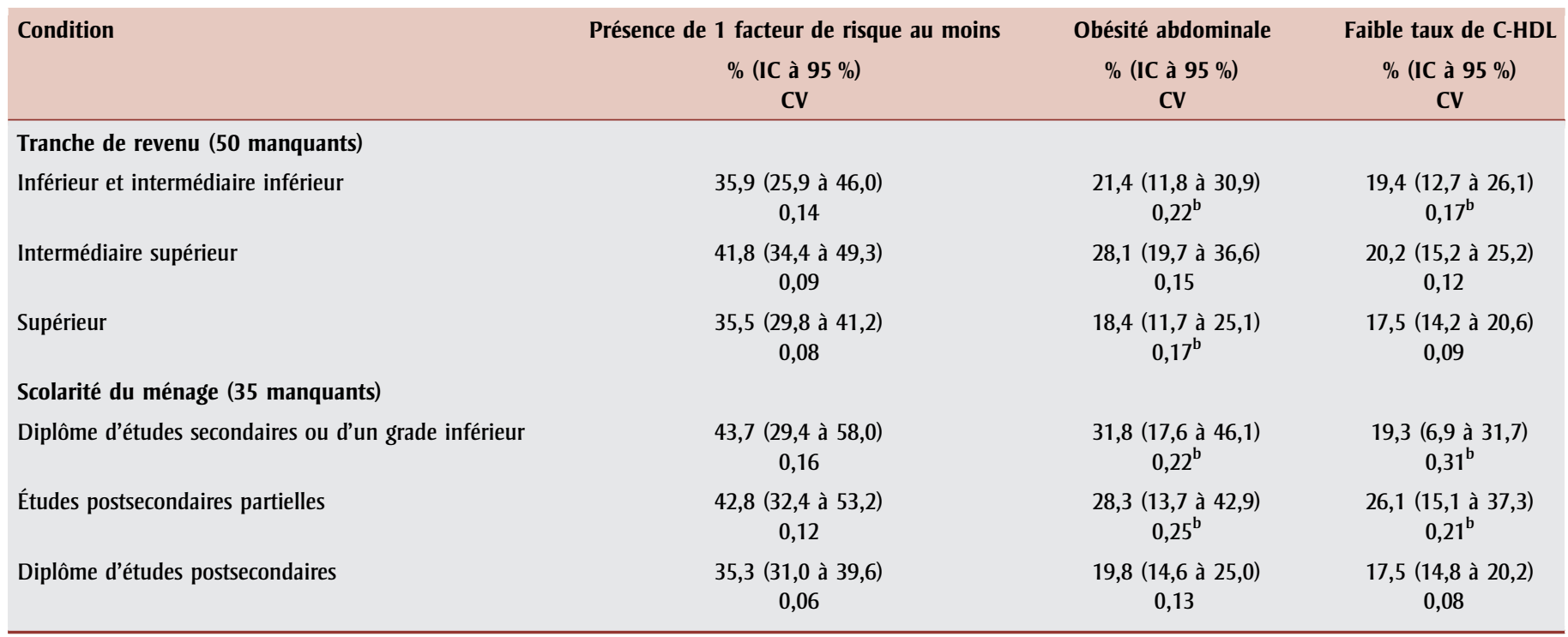

Abréviations : C-HDL, cholestérol à lipoprotéines de haute densité; IC, intervalle de confiance; SSÉ, statut socioéconomique; TA, tension artérielle.

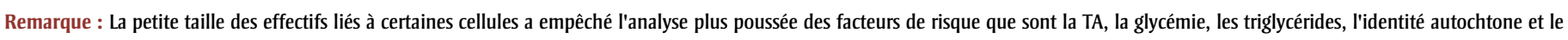
statut vis-à-vis de l'immigration ainsi que des facteurs liés au SSÉ.

a Ces chiffres sont fondés sur des données pondérées.

${ }^{b}$ Ces chiffres sont publiés sous toute réserve car le CV était compris entre 0,16 et 0,33.

Notre estimation d'une plus faible prévalence par rapport à celle rapportée aux ÉtatsUnis est attribuable à plusieurs facteurs. La prévalence du SMet est plus élevée chez les jeunes qui souffrent d'obésité que chez ceux qui ont un poids normal, or la prévalence de l'obésité est plus élevée aux États-Unis qu'au Canada ${ }^{52-54}$. De plus, les estimations de prévalence aux États-Unis ont été établies à l'aide de données tirées de la National Health and Nutrition Examination Survey, qui a connu des variations dans les périodes de collecte de données (entre 1988 et 2006), dans la définition du SMet (variantes de l'ATP III) et dans les critères d'obésité abdominale. Dans le cadre de notre étude, nous avons appliqué rigoureusement la définition du SMet proposée par la FID, en particulier les seuils spécifiques à l'âge et au sexe. De plus, nous avons exclu de notre étude les résidents canadiens habitant dans des réserves ou dans d'autres établissements autochtones, où les populations connaissent une prévalence plus élevée de SMet ${ }^{55-57}$.

Quoique la prévalence du SMet soit globalement faible, il faut souligner que le tiers $(37,7 \%)$ des répondants de l'étude présentaient au moins l'un des facteurs de risque du SMet. Cette constatation, couplée au fait que la prévalence de l'obésité soit de presque $10 \%$ chez les jeunes Canadiens, est inquiétante car les risques de SMet augmentent en présence d'obésité ${ }^{26}$. De plus, étant donné que l'âge est l'un des plus importants prédicteurs du SMet, il est raisonnable de supposer que les enfants et les adolescents qui présentent aujourd'hui un ou plusieurs facteurs de risque sont plus susceptibles de souffrir de SMet plus tard et, de ce fait, de développer des maladies chroniques à l'âge adulte ${ }^{2-4}$. Les données probantes indiquent qu'à long terme, les adultes souffrant de SMet présentent un risque accru de décès attribuable à une MCV, quoiqu'une capacité cardiorespiratoire modérée à élevée atténue en partie ce risque $\mathrm{e}^{53,58}$.

Nos conclusions confirment celles d'autres études selon lesquelles une obésité abdominale, un faible taux de C-HDL et un taux élevé de triglycérides sont les facteurs de risque les plus répandus du SMet chez les enfants et les adolescents ${ }^{28}$ : cette combinaison a compté pour $61,5 \%$ des cas de SMet décelés par l'étude. Le facteur de risque le plus répandu s'est révélé être l'obésité abdominale (21,6 \%), ce qui est attribuable au fait que plus du quart des jeunes Canadiens sont en surpoids ou souffrent d'obésité ${ }^{59}$. La FID fait de l'obésité abdominale une condition de diagnostic de SMet car celle-ci est associée à un risque accru de maladie cardiovasculaire et qu'elle est un prédicteur indépendant d'insulinorésistance, de taux élevés de lipides et d'hypertension artérielle $35,36,60$. Conformément à d'autres études sur les jeunes, la prévalence de l'hypertension ne s'est pas révélée très élevée dans les premiers stades d'apparition du syndrome $\mathrm{e}^{28}$. Nous avons défini l'obésité abdominale au moyen des données de référence en fonction de l'âge et du sexe de l'Enquête sur la condition physique au Canada de 1981 (90 centile $)^{43}$. Or les estimations de la prévalence de l'obésité chez les jeunes à l'échelle nationale ont pratiquement doublé au cours des 25 dernières années, ce qui signifie que ces seuils prédéfinis constituaient la norme pour la population canadienne avant cet important accroissement de la masse adipeuse chez les individus $^{59,61}$.

Les répondants des ménages relevant de revenu et de scolarité supérieurs étaient ceux qui présentaient les plus faibles prévalences d'un ou plusieurs facteurs de risque, d'obésité abdominale et de faible taux de C-HDL, ce qui cadre avec les conclusions d'études antérieures sur les liens entre SSÉ et facteurs de risques du SMet ${ }^{17,19,20,62}$. En ce qui 
concerne l'obésité abdominale, on a pu constater une relation dose-réponse à la fois pour le niveau de scolarité et pour le revenu associés au ménage. La relation entre prévalence des facteurs de risque et niveau de scolarité associé au ménage a semblé être plus sensible que celle liée au revenu du ménage, ce qui cadre également avec les conclusions d'études antérieures ${ }^{17,20,62}$. Cela est attribuable à l'influence de la scolarité sur la littératie et sur les comportements en matière de santé, par exemple concernant la nutrition et l'activité physique, qui sont liés à l'obésité abdominale et au $\mathrm{SMet}^{22,63}$. De plus, à long terme, le niveau de scolarité associé au ménage est considéré comme plus stable et moins influencé par l'état de santé que le revenu du ménage ${ }^{30}$. De façon plus générale, les répondants membres de ménages à moindres niveau de scolarité et revenu sont davantage susceptibles de vivre dans des environnements défavorables sur les plans social, physique et économique, ce qui peut déboucher sur de moins bons résultats en matière de santé, en particulier un taux accru de mortalité attribuable aux $\mathrm{MCV}^{30,47}$. Ces résultats illustrent la nécessité d'interventions, notamment en matière de politiques publiques, d'éducation du public, de recherche et de soins de santé, visant à atténuer les répercussions d'une faible scolarité et d'un faible revenu sur les résultats en matière de santé. Pour que ces interventions soient efficaces et bien ciblées, il serait utile que des travaux de recherche soient consacrés à élucider les enchaînements de causalité par lesquels le SSÉ a une influence sur les risques associés au SMet et aux MCV tout au long de la vie.

Les études qui exploiteront de nouveaux cycles de données de l'ECMS auront sans doute la capacité statistique d'étudier plus en détail le SMet et ses facteurs de risque chez les enfants et les adolescents canadiens. Il conviendrait en particulier d'étudier les différences entre les sexes quant au SMet en fonction du SSÉ, afin de mieux comprendre en quoi le genre affecte les résultats relatifs au SMet dans un contexte socioéconomique défavorable. De plus, on a besoin d'analyses de régression pour examiner en profondeur le lien entre le SMet, ses facteurs de risque, certains comportements (comme l'activité physique et le sommeil) et le SSÉ.

\section{Forces}

Cette étude est la première étude à l'échelle du Canada à appliquer la définition consensuelle du SMet pour les enfants et les adolescents proposée par la FID et à utiliser des données de référence sur le tour de taille des Canadiens en fonction de l'âge et du sexe pour mesurer l'obésité abdominale chez les enfants et les jeunes. Le fait d'appliquer de manière rigoureuse les critères de la FID pour l'étude du SMet à l'échelle de la population du Canada va permettre une comparaison plus fiable avec les futures études du SMet chez les enfants et les adolescents.

Cette étude a été réalisée au moyen de données d'enquêtes publiques de grande qualité et représentatives de $96 \%$ de la population canadienne. Elle souligne plusieurs faits importants sur la santé et l'environnement socioéconomique des enfants et des adolescents canadiens.

\section{Limites}

L'utilisation de statistiques descriptives a été le seul moyen d'étudier le SMet avec ces données sur les enfants et les adolescents canadiens, car l'échantillon était réduit : seuls les répondants ayant fourni des échantillons de sang à jeun ont été sélectionnés. De plus, la faible prévalence du SMet n'a pas permis l'analyse des liens entre chacun des facteurs de risque et le SMet. La taille réduite de l'échantillon a également empêché une analyse statistique solide de l'influence des caractéristiques individuelles et celles du SSÉ sur le SMet, et elle n'a permis qu'une analyse limitée de l'influence de ces variables sur les facteurs de risque, sans distinction possible en fonction du sexe. Nous n'avons pas non plus pu ventiler les résultats en fonction du sexe, de l'âge, de l'identité autochtone ou du statut vis-à-vis de l'immigration. De plus, la conception transversale de l'ECMS limitant l'inférence sur les schémas de causalité sous-jacents aux relations observées, l'étude n’a porté que sur la prévalence de chacun de facteurs de risque du SMet.

Malgré ces limites, les résultats de l'étude permettent de brosser un meilleur portrait global des risques cardiométaboliques chez les jeunes Canadiens.

\section{Conclusion}

Notre étude, qui a porté sur la prévalence du SMet et de ses facteurs de risque chez les enfants et les adolescents canadiens, a mis en lumière plusieurs spécificités importantes liées à l'environnement socioéconomique et à la santé des enfants et les adolescents canadiens. Elle confirme les conclusions d'études antérieures faisant état d'une faible prévalence du SMet chez les jeunes. Cependant, en montrant qu'un jeune sur trois présente au moins un facteur de risque du SMet, qu'un jeune sur cinq présente une obésité abdominale et qu'un jeune sur cinq a un faible taux de de C-HDL, ces résultats constituent d'importants indicateurs de risques potentiels pour la santé chez les jeunes Canadiens. Prévenir, diagnostiquer et traiter le SMet et ses facteurs de risque est important pour prévenir le diabète de type 2 , les maladies cardiovasculaires et les décès prématurés.

\section{Remerciements}

Nous voudrions remercier Statistique Canada pour la tenue de son Enquête canadienne sur les mesures de la santé ainsi que les répondants canadiens qui ont pris part à l'étude.

Nous n'avons aucun conflit d'intérêts à déclarer.

Nous voudrions également remercier pour leur soutien le Département d'épidémiologie et de médecine sociale de l'Université d'Ottawa ainsi que l'Agence de la santé publique du Canada.

\section{Références}

1. Agence de la santé publique du Canada. Travailler ensemble à l'échelle mondiale: Centre collaborateur canadien de l'Organisation mondiale de la santé (OMS) sur les politiques relatives aux maladies non transmissibles [Internet]. Ottawa (Ont.) : Agence de la santé publique du Canada; 2012 [consulté le $1^{\text {er }}$ février 2014]. Consultable à la page : http://www.phac-aspc.gc.ca/about_ apropos/whocc-ccoms/index-fra.php 
2. Ford ES. Risks for all-cause mortality, cardiovascular disease, and diabetes associated with the metabolic syndrome: a summary of the evidence. Diabetes Care. 2005;28(7): 1769-1778.

3. Isomaa A, Almgren P, Tuomi $\mathrm{T}$ et collab. Cardiovascular morbidity and mortality associated with the metabolic syndrome. Diabetes Care. 2001;24(4):683-689.

4. McNeill AM, Rosamond WD, Girman CJ et collab. The metabolic syndrome and 11-year risk of incident cardiovascular disease in the Atherosclerosis Risk in Communities study. Diabetes Care. 2005;28(2):385-390.

5. Haffner SM, Valdex RA, Hazuda HP, Mitchell BD, Morales PA, Stern MP. Prospective analysis of the insulin resistance syndrome (Syndrome X). Diabetes. 1992; 41(6):715-722.

6. Alberti KG, Zimmet PZ, Shaw J. Metabolic syndrome - a new world-wide definition. Lancet. 2005;366(9491):1059-1062.

7. Huang TT, Ball GD, Franks PW. Metabolic syndrome in youth: current issues and challenges. Appl Physiol Nutr Metab. 2007;32(1):13-22.

8. Haffner SM. The metabolic syndrome: inflammation, diabetes mellitus, and cardiovascular disease. Am J Cardiol. 2006; 97(2A):3A-11A.

9. Alberti KG, Zimmet PZ. Definition, diagnosis and classification of diabetes mellitus and its complications, part 1: provisional report of a WHO consultation. Diabetes Med. 1998;15(7):539-553.

10. Reaven GM. Role of insulin resistance in human disease. Diabetes. 1988;37(12):1595-1607.

11. Dandona P, Aljada A, Chaudhuri A, Mohanty P, Garg R. Metabolic syndrome: a comprehensive perspective based on interactions between obesity, diabetes and inflammation. Circulation. 2005;111(11):1448-1454.

12. Zimmet P, Alberti KG, Shaw J. Global and societal implications of the diabetes epidemic. Nature. 2001;414:782-787.

13. Eckel RH, Grundy SM, Zimmet PZ. The metabolic syndrome. Lancet. 2005;364(9468): 1415-1428.
14. Goodman E, Daniels SR, Morrison JA, Huang B, Dolan LM. Contrasting prevalence of and demographic disparities in the world health organization and national cholesterol education program adult treatment panel III definitions of metabolic syndrome among adolescents. J Pediatr. 2004;4(59):445-451.

15. Tailor AM, Peeters PH, Norat T, Vineis P, Romaquera D. An update on the prevalence of the metabolic syndrome in children and adolescents. Int J Pediatr Obes. 2010;5(3): 202-213.

16. Cameron AJ, Shaw JE, Zimmet PZ. The metabolic syndrome: prevalence in worldwide populations. Endocrinol Metab Clin North Am. 2004;33(2):351-375.

17. Riediger ND, Clara I. Prevalence of metabolic syndrome in the Canadian adult population. CMAJ. 2011;183(15):E1127-E1134.

18. Statistique Canada. Feuillets d'information sur la santé - Syndrome métabolique chez les Canadiens, 2009 à 2011. Ottawa (Ont.) : Statistique Canada; 2012. (Statistique Canada, $\mathrm{n}^{\circ}$ 82-625-X au catalogue)

19. Setayeshgar S, Whiting SJ, Vatanparast H. Metabolic syndrome in Canadian adults and adolescents: prevalence and associated dietary intake. ISRN Obesity. 2012;2012:1-8.

20. Ardern CI, Katzmarzyk PT. Geographic and demographic variation in the prevalence of the metabolic syndrome in Canada. Can J Diabetes. 2007;31(1):34-46.

21. Brenner D, Arora P, Karmali M, Badawi A. The impact of the metabolic syndrome on cardiometabolic and inflammatory profiles among Canadian adults. J Epidemiol Community Health. 2011;65(A):A228-A229.

22. Brien SE, Katzmarzyk PT. Physical activity and the metabolic syndrome in Canada. Appl Physiol Nutr Metab. 2006;31(1):40-47.

23. Ford ES, Giles WH, Mokdad AH. Increasing prevalence of the metabolic syndrome among US adults. Diabetes Care. 2004; 27(10):2444-2449.

24. Ervin RB. Prevalence of metabolic syndrome among adults 20 years of age and over, by sex, age, race and ethnicity, and body mass index: United States, 2003-2006. Natl Health Stat Report. 2009;13:1-7.
25. Ford ES, Giles WH, Dietz WH. Prevalence of the metabolic syndrome among US adults: findings from the third National Health and Nutrition Examination Survey. JAMA. 2002;287(3):356-359.

26. Park YW, Zhu S, Palaniappan L, Heshka S, Carnethon MR, Heymsfield SB. The metabolic syndrome: prevalence and associated risk factor findings in the US population from the third National Health and Nutrition Examination Survey, 19881994. Arch Intern Med. 2003;163(4): 427-436.

27. De Ferranti SD, Gauvreau K, Ludwig DR, Neufeld EJ, Newburger J, Rifai N. Prevalence of the metabolic syndrome in American adolescents: findings from the third National Health and Nutrition Examination Survey. Circulation. 2004;110(16) 2494-2497.

28. Johnson WD, Kroon JJ, Greenway FL, Bouchard C, Ryan D, Katzmarzyk PT. Prevalence of risk factors for metabolic syndrome in adolescents: National Health and Nutrition Examination Survey (2001-2006). Arch Pediatr Adolesc Med. 2009;163(4):371-377.

29. Cook S, Weitzman M, Auinger P, Nguyen M, Dietz WH. Prevalence of a metabolic syndrome phenotype in adolescents: findings from the third National Health and Nutrition Examination Survey, 1988-1994. Arch Pediatr Adolesc Med. 2003;157(8): 821-827.

30. Kaplan GA, Julian E. Socioeconomic factors and cardiovascular disease: a review of the literature. Circulation. 1993;88(4):1973-1998.

31. Marmot M. Income inequality, social environment, and inequalities in health. J Policy Anal Manage. 2001;20(1):156-159.

32. Hemingway $H$, Shipley $M$, Macfarlane $P$, Marmot M. Impact of socioeconomic status on coronary mortality in people with symptoms, electrocardiographic abnormalities, both or neither: the original Whitehall study 25 year follow up. J Epidemiol Community Health. 2000;54(7):510-516.

33. Brunner EJ, Marmot MG, Nanchahal K et collab. Social inequality in coronary risk: central obesity and the metabolic syndrome, evidence from the Whitehall II study. Diabetologia. 1997;40(11):1341-1349. 
34. Santos AC, Ebrahim S, Barros H. Gender, socio-economic status and metabolic syndrome in middle-aged and old adults. BMC Public Health. 2008;8:62.

35. International Diabetes Federation. The IDF consensus worldwide definition of the metabolic syndrome [Internet]. Bruxelles : IDF Communications; 2006. PDF téléchargeable à partir du lien : http://www.idf. org/webdata/docs/IDF_Meta_def_final.pdf

36. International Diabetes Federation. The IDF consensus definition of the metabolic syndrome in children and adolescents [Internet]. Bruxelles : IDF Communications; 2007. PDF téléchargeable à partir du lien : http:// www.idf.org/webdata/docs/Mets_definition_children.pdf

37. Balkau B, Charles MA. Comment on the provisional report from the WHO consultation, European Group for the Study of Insulin Resistance. Diabetes Med. 1999; 16(5):442-443.

38. National Heart Lung and Blood Institute, National Cholesterol Education Program Expert Panel on Detection Evaluation and Treatment of High Blood Cholesterol in Adults. Third report of the National Cholesterol Education Program Expert Panel on detection, evaluation, and treatment of high blood cholesterol in adults (Adult Treatment Panel III). Circulation. 2002;106(25): 3143-3421.

39. Statistique Canada. Guide de l'utilisateur des données de l'Enquête canadienne sur les mesures de la santé (ECMS) : cycle 2 [Internet]. Ottawa (Ont.) : Statistique Canada; 2013 [consulté le $1^{\text {er }}$ octobre 2013]. Consultable à la page :http://www23.statcan.gc.ca/imdb-bmdi/ document/5071_D2_T1_V2-fra.htm

40. Statistique Canada. Guide de l'utilisateur des données de l'Enquête canadienne sur les mesures de la santé (ECMS) : cycle 1 [Internet]. Ottawa (Ont.) : Statistique Canada; 2011 [consulté le $1^{\mathrm{er}}$ octobre 2013]. Consultable à la page : http://www23.statcan.gc.ca/imdbbmdi/document/5071_D2_T1_V1-fra.htm

41. Tremblay MS, Connor Gorber SC. Canadian Health Measures Survey: brief overview. Can J Public Health. 2007;98(6):453-456.

42. Giroux S. Enquête canadienne sur les mesures de la santé : aperçu de la stratégie d'échantillonnage. Rapports sur la santé, 2007;18, Supplément, 35-40.
43. Katzmarzyk PT. Waist circumference percentiles for Canadian youth 11-18 years of age. Eur J Clin Nutr. 2004;58(7):1011-1015.

44. Winkleby MA, Jatulis DE, Frank E, Fortmann SP. Socioeconomic status and health: how education, income, and occupation contribute to risk factors for cardiovascular disease. Am J Public Health. 1992;82(6):816-820.

45. Liberatos P, Link BG, Kelsey JL. The measurement of social class in epidemiology. Epidemiol Rev. 1988;10(1):87-121.

46. Duncan GJ, Daly MC, McDonough P, Williams DR. Optimal Indicators of socioeconomic status for health research. Am J Public Health. 2002;92(7):1151-1157.

47. Organisation mondiale de la santé, Commission des déterminants sociaux de la santé. Combler le fossé en une génération : instaurer l'équité en santé en agissant sur les déterminants sociaux - Rapport final. Genève $(\mathrm{CH})$ : Organisation mondiale de la santé; 2008.

48. McIntosh $\mathrm{CN}$, Finès $\mathrm{P}$, Wilkins $\mathrm{R}$, Wolfson MC. Disparités selon le revenu dans l'espérance de vie ajustée sur la santé chez les adultes au Canada, 1991 à 2001. Rapports sur la santé. 2009;20(4):59-70.

49. Efron B, Tibshirani R. Bootstrap methods for standard errors, confidence intervals, and other measures of statistical accuracy. Statist Sci. 1986 Feb; 1(2):54-75.

50. Jolliffe CJ, Janssen I. Development of agespecific adolescent metabolic syndrome criteria that are linked to the Adult Treatment Panel III and International Diabetes Federation criteria. J Am Coll Cardiol. 2007;49(8):891-898.

51. Kassi E, Pervanidou P, Kaltsas G, Chrousos G. Metabolic syndrome: definitions and controversies. BMC Med. 2011;9:48.

52. Weiss R, Dziura J, Burgert TS et collab. Obesity and the metabolic syndrome in children and adolescents. New Engl J Med. 2004;350:2362-2374.

53. Katzmarzyk PT, Church TS, Janssen I, Ross R, Blair SN. Metabolic syndrome, obesity, and mortality: impact of cardiorespiratory fitness. Diabetes Care. 2005;28(2):391-397.

54. Lee S, Bacha F, Arslanian SA. Waist circumference, blood pressure, and lipid components of the metabolic syndrome. J Pediatr. 2006;49(6):809-816.
55. Pollex RL, Hanley AJ, Zinman, Harris SB, Khan HM, Hegele RA. Metabolic syndrome in Aboriginal Canadians: prevalence and genetic association. Atherosclerosis. 2006;184(1):121-129.

56. Liu J, Young TK, Zinman B, Harris SB, Connelly PW, Hanley AJ. Lifestyle variables, non-traditional cardiovascular risk factors, and the metabolic syndrome in an Aboriginal Canadian population. Obesity (Silver Spring). 2005;14(3):500-508.

57. Kaler SN, Ralph-Campbell K, Pohar S, King M, Laboucan CR, Toth EL. High rates of the metabolic syndrome in a First Nations community in western Canada: prevalence and determinants in adults and children. Int J Circumpolar Health. 2006;65(5):389-402.

58. Hunt KJ, Resendez RG, William KW, Haffner SM, Stern MP. National Cholesterol Education Program versus World Health Organization metabolic syndrome in relation to all-cause and cardiovascular mortality in the San Antonio Heart Study. Circulation. 2004;110:1251-1257.

59. Tremblay MS, Wilms JD. Secular trends in the body mass index of Canadian children. CMAJ. 2000;163(11):1429-1433.

60. Burke V, Beilin LJ, Simmer K et collab. Predictors of body mass index and associations with cardiovascular risk factors in Australian children: a prospective cohort study. Int J Obesity (Lond). 2005;29:15-23.

61. Agence de la santé publique du Canada; Institut canadien d'information sur la santé. Obésité au Canada : rapport conjoint de l'Agence de la santé publique du Canada et de l'Institut canadien d'information sur la santé. Ottawa (Ont.) : Agence de la santé publique du Canada; 2011.

62. Loucks EB, Rehkopf DH, Thurston RC, Kawachi I. Socioeconomic disparities in metabolic syndrome differ by gender: evidence from NHANES III. Ann Epidemiol. 2007;17(1):19-26.

63. Dallongeville J, Cottel D, Ferrières J et collab. Household income is associated with the risk of metabolic syndrome in a sex-specific manner. Diabetes Care. 2005;28(2):409-415. 